prognostic factors for a good outcome are available, although mechanical thrombectomy has significantly advanced over the last 5 years. The aim of this study is to investigate good prognostic factors for an acute occlusion of a major cerebral artery using mechanical thrombectomy.

Methods A single center retrospective analysis of 37 consecutive patients with acute occlusion of a major cerebral artery treated by mechanical thrombectomy with stent retrievers was conducted. Collaterals were assessed by the Thrombolysis in Myocardial Infarction (TIMI), and recanalization was assessed by the Thrombolysis in Cerebral Infarction (TICI) score. Outcome was assessed by National Institutes of Health Stroke Scale (NIHSS) and modified Rankin Scale (mRS) at 90 days.

Results Most patients (27/37) demonstrated good recanalization (TICI $2 \mathrm{~b}$ or 3) after thrombectomy. At the 90-day follow up, 19 patients had good (mRS, 0-2), 14 had moderate (mRS, $3-4)$ and four had poor outcomes (mRS, 5-6). Early recanalization, high TIMI, and low baseline NIHSS were closely related to 90-day mRS, whereas high TICI was related to both mRS and the decrease in the NIHSS.

Conclusions NIHSS decreased markedly when recanalization was successful. A good mRS was related to low initial NIHSS and good collateral and early and successful recanalization.

Disclosures S. Park: None.

\section{E-035 SUPERIOR OUTCOMES IN THROMBECTOMY FOR ANTERIOR CIRCULATION LARGE VESSEL OCCLUSION STROKE AMONG LATE MR-SELECTED CANDIDATES COMPARED WITH EARLY CANDIDATES NOT SCREENED WITH MR}

B Cristiano, M Pond, U Oyoyo, S Basu, J Jacobson. Neuroradiology, Loma Linda University Hospital, Loma Linda, CA

\subsection{6/neurintsurg-2016-012589.107}

Purpose With thrombectomy for anterior circulation large vessel occlusion (ACLVO) stroke time is considered important, but collateral status may be a greater driver of outcome than time. MR screening can identify good candidates for thrombectomy regardless of time from onset. Here we tested the hypothesis that MR-selected patients would show superior outcomes regardless of time compared with early patients screened by CTA alone.

Methods A cohort of 56 ACLVO patients treated with thrombectomy between $11 / 1 / 2012$ and $5 / 15 / 2015$ was retrospectively studied. Seven early-presenting patients with contraindication to MRI proceeded immediately to thrombectomy upon CTA confirmation of LVO. Forty-nine patients with CTA-proven LVO but no MR contraindication went next to MRI and were selected for thrombectomy based on low diffusionrestricted infarct volume (non-intervention threshold: core volume $>$ (100 minus patient age) $\mathrm{mL}$ ). Final infarct volume was measured on post-treatment imaging. Comparisons were made between early CTA-only-screened and MR-screened groups. Additional comparisons were made with the late MR-screened (decision-to-treat $>6$ hours from symptom onset) subset.

Results Compared to the MR-screened group, the early CTAonly group had a higher median age (81 [IQR 76-83] vs. 71 [57-77]) and NIHSS score (25 [22-26] v. 15 [11-19]) was more likely to have received IV tPA $(71.4 \%$ vs. $36.7 \%)$ and yielded a lower TICI $\geq 2 \mathrm{~B}$ recanalization rate $(57.1 \%$ vs. 79.6\%, $p=0.36$ ). Despite significantly earlier treatment decision times $(3.0 \mathrm{~h}$ [2.5-3.6] vs. $6.4 \mathrm{~h}$ [2.9-8.7]), median final infarct volume was larger in the early CTA-only group compared with the MR-selected group $(134 \mathrm{~mL}$ vs. $20 \mathrm{~mL}$, estimated median final infarct difference of $100 \mathrm{~mL}$ [95 CI: 1.00-134], $p=0.043$ ). A similar difference in median final infarct volume was observed between CTA-only and the late MR-screened groups (134 mL v. $27 \mathrm{~mL}$ [95 CI: -6 - 132], $p=0.069$ ) (Figure 1). The odds of a final core infarct greater than $70 \mathrm{~mL}$, a poor prognostic factor, were significantly lower in the MR-screened group OR 0.013 ([95 CI: 0.022-0.757], $p=0.022$ ).

Conclusion Despite a median 3.4 hour-shorter interval from symptom onset to treatment decision, ACLVO patients not screened with MRI had larger median final infarct volumes after thrombectomy compared with MR-screened patients. Importantly, late MR-selected patients also tended to have lower final infarct volumes. Consideration should be given to patient selection strategies incorporating MRI with diffusion weighted sequences rather than time from symptom onset alone.


Abstract E-035 Figure 1 Final Infarct volumes in CTA-only versus MR-screened adfter thrombectomy. Median final core volume $(\mathrm{mL})$ : CTA-Only, 134; MR-screened, 20; late MR-screened, 27. Proportion of patients with large final core volume (>70 mL): CTA-only, 71.4\%; MRscreened, 23.1\%; late MR-screened, 26.1\%. Dashed line indicates poor outcome final infaract threshold of $70 \mathrm{~mL}$, bars indicate median and IQR.

Disclosures B. Cristiano: None. M. Pond: None. U. Oyoyo: None. S. Basu: None. J. Jacobson: 4; C; GeneLux.

\section{E-036 PREDICTING FLOW DIVERTER DEPLOYMENTS AND CLINICAL VALIDATION}

${ }^{1}$ B Chong, ${ }^{2} \mathrm{H}$ Babiker, ${ }^{3} \mathrm{Y}$ Kalani, ${ }^{4} \mathrm{C}$ Baccin, ${ }^{2} \mathrm{M}$ Mortensen, ${ }^{3} \mathrm{M}$ Levitt, ${ }^{3} \mathrm{C}$ McDougall, ${ }^{5} \mathrm{D}$ Frakes, ${ }^{3} \mathrm{~F}$ Albuquerque. ${ }^{1}$ Radiology and Neurological Surgery, Mayo Clinic Arizona, Phoenix, AZ; ${ }^{2}$ Endovantage, LLC, Scottsdale, AZ; ${ }^{3}$ Neurological Surgery, Barrow Neurological Institute, Phoenix, AZ; ${ }^{4}$ Radiology, Hospital Israelita Albert Einstein, Sao Paulo, Brazil; ${ }^{5}$ School of Biological and Health Systems Engineering, Arizona State University, Phoenix, AZ

10.1136/neurintsurg-2016-012589.108 